\title{
Ginsenoside Rg1 and Resveratrol Alleviate Acute Kidney Injury Induced by Cisplatin via Downregulation of Autophagy in Mice
}

\author{
Yu Liu1*, Jiao Qiu1*, Ruiqiao Tan1*, Qing Tian², Li Guan², Shuaishuai Niu², Sijia Huang2, \\ Jing Huang2, Yunbo Yan², Ying Xiang1,2\# \\ ${ }^{1}$ Laboratory of Oncology, Center for Molecular Medicine, School of Basic Medicine, Health Science Center, Yangtze University, \\ Jingzhou, China \\ ${ }^{2}$ Department of Cell Biology and Genetics, School of Basic Medicine, Health Science Center, Yangtze University, Jingzhou, China \\ Email: "xying316@163.com
}

How to cite this paper: Liu, Y., Qiu, J., Tan, R.Q., Tian, Q., Guan, L., Niu, S.S., Huang, S.J., Huang, J., Yan, Y.B. and Xiang, Y. (2021) Ginsenoside Rg1 and Resveratrol Alleviate Acute Kidney Injury Induced by Cisplatin via Downregulation of Autophagy in Mice. Yangtze Medicine, 5, 12-22. https://doi.org/10.4236/ym.2021.51002

Received: February 3, 2020

Accepted: January 5, 2021

Published: January 8, 2021

Copyright $\odot 2021$ by author(s) and Scientific Research Publishing Inc. This work is licensed under the Creative Commons Attribution International License (CC BY 4.0).

http://creativecommons.org/licenses/by/4.0/

(c) $\underset{\mathrm{BV}}{\mathrm{BV}}$ Open Access

\begin{abstract}
Background: Cisplatin, a chemotherapeutic agent, is widely used in the treatment of malignant tumors. Nephrotoxicity, especially acute kidney injury (AKI), is the most common and severe adverse reaction of cisplatin. Resveratrol and ginsenoside $\mathrm{Rg} 1$, two natural products, have been found to have renal protective effects. However, the effects and the mechanisms in cisplatin-induced AKI need further investigation. Methods: The mouse models of cisplatin-induced AKI and several treatment groups were established. Male C57BL/6 mice were divided into five groups: saline control group, cisplatin injury group, resveratrol treatment group, $\mathrm{Rg} 1$ treatment group, resveratrol and Rg1 combined treatment group. Serological analysis of serum urea nitrogen was aimed to reflect the function of kidney, and histological analysis of renal tissue sections was aimed to assess the damage of proximal convoluted tubules. The expression levels of autophagy-related proteins Beclin 1 and LC3 were detected by western blotting and qRT-PCR respectively. Results: The renal function was improved and renal damage was alleviated in Rg1 and resveratrol alone or combined treatment groups compared with the cisplatin injury group. For the mechanism, treatment with Rg1 and resveratrol alone or in combination decreased the expressions of Beclin 1 both at protein and mRNA levels, decreased LC3II/I protein levels, indicating that autophagy was inhibited by treatment with $\mathrm{Rg} 1$ and resveratrol alone or in combination. Conclusion: Resveratrol and Rg1 alleviated the kidney damage caused by cisplatin, and reduced autophagy was involved in the renoprotective effects of
\end{abstract}


resveratrol and Rg1 against cisplatin-induced AKI. This study may provide new evidence to alleviate cisplatin-induced AKI.

\section{Keywords}

Cisplatin, Acute Kidney Injury, Resveratrol, Ginsenoside Rg1, Autophagy

\section{Introduction}

Cisplatin, a chemotherapeutic drug, is widely used in the treatment of malignant tumors. Nephrotoxicity is the most common adverse reaction of cisplatin and the main dose limiting factor in cisplatin therapy. There are many manifestations of cisplatin nephrotoxicity, among which the most serious and common manifestation is acute kidney injury (AKI) [1]. Cisplatin nephrotoxicity can lead to renal tubular cell injury and death, severe inflammation, renal ischemia, acute renal failure and a series of serious consequences. Although diuretics, hydration and dosage adjustment can be used to control AKI, the incidence of AKI is still very high [2]. Clinically, cisplatin is often used in combination with drugs to protect renal function to improve the renal injury induced by cisplatin. Therefore, it is of great clinical value and significance to explore ideal renoprotective drugs.

Plant extracts with the characteristics of low toxicity, wide source of raw materials and low cost, are potential ideal renal protective drugs. Resveratrol and ginsenoside Rg1 are two important natural products. In recent years, studies have shown that resveratrol can ameliorate kidney damage caused by various causes, including renal injury by cisplatin in animal experiments [3], and reducing cytotoxicity of proximal renal tubules induced by cisplatin [4]. Ginsenoside Rg1 has been shown to ameliorate renal failure [5], diabetic nephropathy [6]. In summary, we hypothesize that resveratrol combined with ginsenoside Rg1 may play a more effective protective role in cisplatin-induced AKI.

Autophagy is a process of degradation of damaged organelles, protein aggregates and other macromolecules in the cytoplasm. The role of autophagy in renal injury is controversial. It has been found that autophagy is an important protective mechanism for cell survival in acute kidney injury [7]. Jiang et al. showed that autophagy had a protective effect on AKI [8]. However, studies have shown that during the recovery of renal injury, autophagy can aggravate tubular dysfunction [9]. Therefore, we proposed that the effect of autophagy on renal injury may be related to the time of stimulation.

In this study, we investigated the role of autophagy-related protein Beclin 1 and LC3 in renoprotective effect of Rg1 and resveratrol against cisplatin-induced $\mathrm{AKI}$ in mice. It was aimed to reveal the mechanism of $\mathrm{Rg} 1$ and resveratrol protected the kidney and provide evidence on the therapeutic application in alleviating AKI. 


\section{Materials and Methods}

\section{Drugs}

Cisplatin, ginsenoside Rg1, resveratrol were purchased from Meilun Biotech (Dalian, China). Cisplatin was dissolved in saline at $1 \mathrm{mg} / \mathrm{ml}$, resveratrol was dissolved in saline with $1 \% \mathrm{DMSO}$ at $2 \mathrm{mg} / \mathrm{ml}$, and $\mathrm{Rg} 1$ was dissolved in saline at $2 \mathrm{mg} / \mathrm{ml}$.

\section{Mouse model of cisplatin-induced $A K I$ and treatments}

C57BL/6 male mice, 8 - 10 weeks old, weighing $20-25 \mathrm{~g}$, were purchased from the Experimental Animal Center of the Three Gorges University (No. 2017-0012, Hubei, China). The mice were allowed to acclimate for one week before experiments, caged in a room with suitable temperature and illumination, and free to access food and water. Then the mice were divided into five groups: saline control group (Control), cisplatin injury group (CP), resveratrol treatment group (RES), $\mathrm{Rg} 1$ treatment group ( $\mathrm{Rg} 1$ ), resveratrol and $\mathrm{Rg} 1$ combined treatment group (RES + Rg1). The cisplatin group received a single injection with cisplatin $(20 \mathrm{mg} / \mathrm{kg})$. The control group received injection with equal-volume saline. The resveratrol treatment group received i.p. RES (30 mg/kg/day) for three days before injection with cisplatin $(20 \mathrm{mg} / \mathrm{kg})$, and continued another three days until the day before scarification. The $\mathrm{Rg} 1$ treatment group received i.p. Rg1 (20 mg/kg/day) for three days before injection with cisplatin $(20 \mathrm{mg} / \mathrm{kg})$, and continued another three days until the day before scarification. The resveratrol and Rg1 combined treatment group received i.p. RES (30 mg/kg/day) and Rg1 (20 mg/kg/day) for three days before injection with cisplatin $(20 \mathrm{mg} / \mathrm{kg})$, and continued for another three days until the day before scarification. The cisplatin injury group (including 18 mice) was sacrificed at different time-point after cisplatin treatment. The other groups (at least 7 mice for each group) were sacrificed three days after cisplatin treatment. All animal experiments were conducted at the Animal Center of Yangtze University, accredited by the Association for Assessment and Accreditation of Laboratory Animal Care International (AAALAC International), in accordance with the Guidelines for the Care and Use of Laboratory Animals.

\section{Serological analysis}

Blood urea nitrogen (BUN) diagnostic kit was purchased from Nanjing Jiancheng Bioengineering Institute (China). Mouse blood was collected in a $1.5 \mathrm{ml}$ centrifuge tube, then placed at $37^{\circ} \mathrm{C}$ for $2-4$ hours, and then centrifuged at 3000 rpm for 15 minutes to separate the serum. Serum BUN was tested according to the BUN kit instructions.

\section{Histological analysis}

After the mice were sacrificed, the kidneys were carefully separated. One kidney of each mouse was subjected to histological analysis, the other was subjected to extraction of the total RNA and proteins. Kidneys were washed with PBS and fixed with $4 \%$ paraformaldehyde. After dehydrated, embedded, sliced, the tissues were stained with hematoxylin-eosin (HE) solution. The nucleus was stained 
purple-blue, and the components in the cytoplasm and extracellular matrix were stained red. Then the general morphology characteristics of kidney tissues were observed under light microscope.

\section{Quantitative Real-time PCR ( $q R T-P C R)$}

The total RNA was extracted from kidney tissues using TRIzol reagent (Beyotime, China). Reverse transcribed complementary DNA was synthesized using random primers. Subsequently, real-time PCR was performed on a 7500 real-time PCR system. Primers for Beclin 1 and GAPDH were synthesized by Songon Biotech (Shanghai, China). GAPDH was used as an internal control. The primers sequences for Beclin 1: sense 5'-TCCCGAGGTGAAGAGCATCG-3', anti-sense 5'-AGTTCCTGGATGGTGACACG-3'. The primers sequences for GAPDH: sense 5'-GTTGTCTCCTGCGACTTCA-3', anti-sense 5'-GGTGGTCC AGGGTTTCTTA-3'.

\section{Western blotting}

The proteins in renal tissues were extracted with RIPA lysate. Then the proteins were separated by SDS-polyacrylamide gel and electrophoretically transferred to a PDVF membrane. The membrane was blocked with 5\% skim milk powder and incubated with antibodies to GAPDH, Beclin 1 and LC3 (Multi Sciences, China), overnight at $4^{\circ} \mathrm{C}$. After washed with PBST, the membrane was incubated with a horseradish peroxidase-conjugated secondary antibody. Bands were viewed using ECL chemiluminescence (Meilun, China) and quantified with Image J software. GAPDH was used as internal control.

\section{Statistical analysis}

All experiments were conducted at least three independent performances, and all the data were expressed as means $\pm \mathrm{SD}$. ANOVA could be used to determine the statistical importance of differences between groups. Differences were regarded significant at $P<0.05$.

\section{Results}

\section{Rg1 and resveratrol alleviate mouse acute renal injury induced by cispla- tin}

In order to assess the mouse model of acute renal injury, we measured blood urea nitrogen (BUN) of mice. The value of BUN was increased significantly in cisplatin injury group compared with saline control group. BUN values were decreased in Rg1 and resveratrol alone or combined treatment groups, in comparison with cisplatin injury group (Figure 1(a)).

As shown in Figure 1(b), the morphological structure of the kidney in the control group was normal under the light microscope, while the morphological changes in the cisplatin injury group showed varying degrees, which confirmed that cisplatin could cause AKI (Figure 1(b)). Pathological results showed that the damage mainly occurred in the proximal convoluted tubule, and the main manifestations were nuclear disintegration and the decrease in number of cells. In comparison with the cisplatin injury group, the symptoms were significantly 


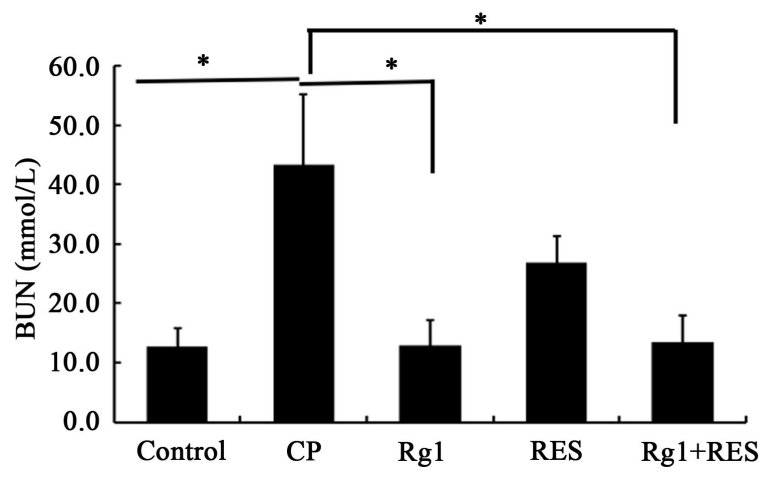

(a)

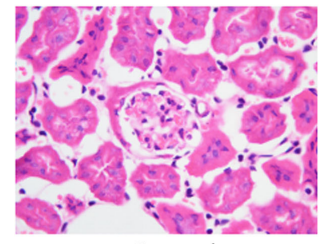

Control

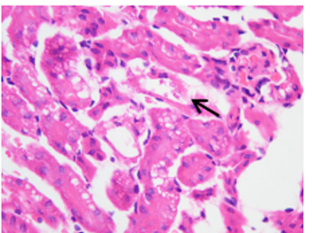

CP

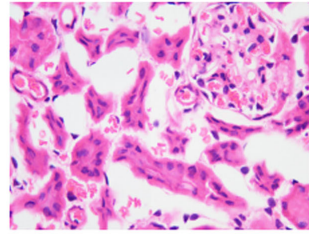

RG1

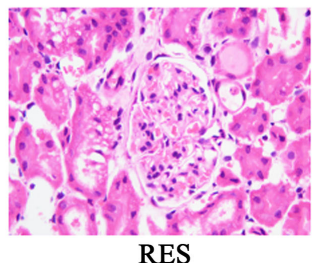

(b)

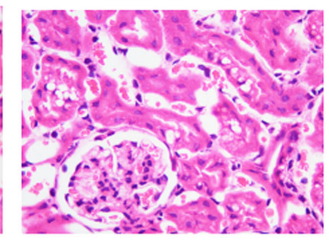

Rg1+RES

Figure 1. Rg1 and resveratrol alleviate mouse acute renal injury induced by cisplatin. Five groups of C57BL/6 mice (7 mice for each group): 1) Control: saline control group, 2) CP: cisplatin injury group, 3) Rg1: cisplatin + Rg1, 4) RES: cisplatin + resveratrol, 5) Rg1 + RES: cisplatin + Rg1 + resveratrol. (a) Peripheral blood was collected from retro-orbital sinus at sacrifice and the values of BUN were measured. Data are expressed as mean \pm SD. ${ }^{*} P<0.05$; (b) HE staining of kidney tissues. The arrow indicates the nuclear disintegration of proximal convoluted tubules.

reduced in the treatment groups with Rg1 and resveratrol alone or in combination. These results suggested that treatment with $\mathrm{Rg} 1$ and resveratrol may alleviate mice acute renal injury induced by cisplatin, and improve kidney function.

\section{Autophagy is induced by cisplatin in renal tissues of mice}

Beclin 1, a member of autophagy-related (Atg) family, which is a key regulatory factor of autophagy and an essential molecule for the formation of autophagosome. As a "platform" for molecular reaction, it can mediate the localization of autophagy-related proteins in phagocytic vesicles and regulate the formation and maturation of autophagosome by reacting with various proteins [10]. Beclin 1 protein can interact with members of the anti-apoptotic proteins of $\mathrm{Bcl}$ family, such as Bcl-2, Bcl-xl and Bcl-w [11]. LC3 has two forms in the cell, namely LC3-I and LC3-II. During autophagy, LC3-I is ubiquitylation and covalently binds with PE to produce LC3-II. LC3-II, located on the membrane of autophagosome, is a specific marker protein of autophagy. Transformation of LC3-I to LC3-II, indicating that autophagic vacuole closure, and the amount of 
LC3-II transformation is related to the degree of autophagosome formation and used to assess the autophagic activity [12].

Western blotting was used to detect the protein levels of Beclin 1 and LC3, and the results showed that both were significantly changed three days after cisplatin injection. The transformation of LC3-I to LC3-II was significantly increased a time-dependent manner, and Beclin 1 expression was also significantly increased in a time-dependent manner, suggesting that autophagy was induced by cisplatin (Figure 2(a) and Figure 2(b)).

Rg1 and resveratrol decrease autophagy in the kidneys of mice intervened with cisplatin

To investigate the mechanism of $\mathrm{Rg} 1$ and resveratrol in renoprotection, we examined the expression levels of autophagy-related proteins Beclin 1 and LC3 in the kidney. We found that the protein levels of Beclin 1 were decreased in the three treatment groups than cisplatin injury group, and the transformation of LC3-I to LC3-II were also reduced (Figure 3(a)). Then, we detected mRNA

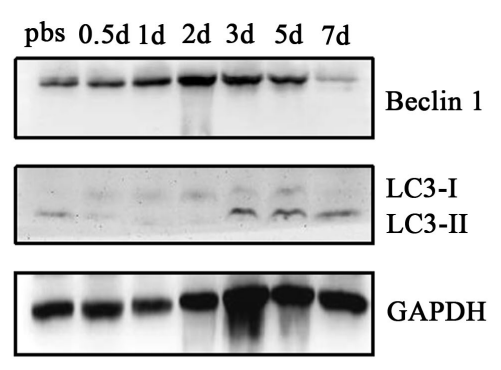

(a)

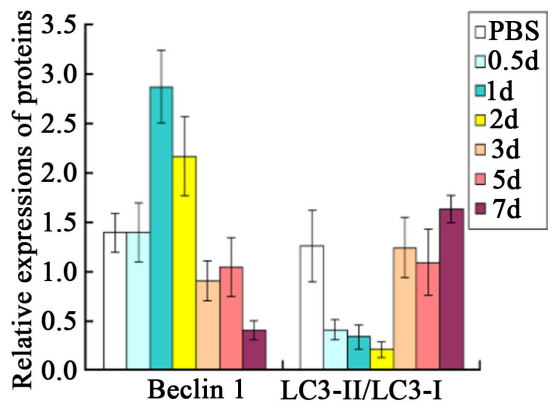

(b)

Figure 2. Autophagy is induced in renal tissues of mice by cisplatin. (a) The expression of Beclin 1 and LC3 of renal tissues were determined by western blotting three days after cisplatin injection. GAPDH served as an internal control; (b) The relative expressions of Beclin 1 and LC3II/I were quantified by Image J software, and data are expressed as mean $\pm \mathrm{SD}$.

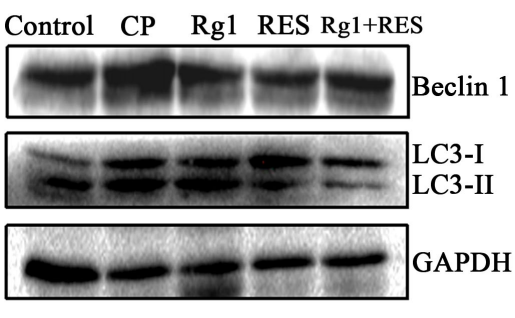

(a)

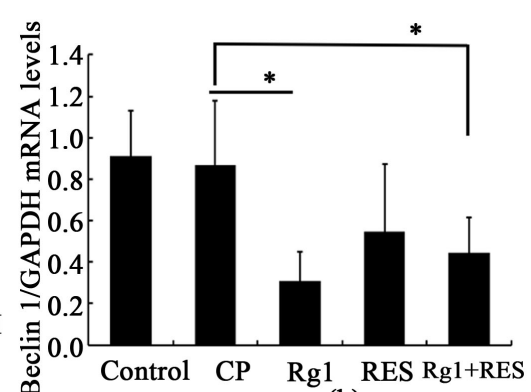

(b)

Figure 3. Rg1 and resveratrol decrease autophagy in the kidneys of mice intervened with cisplatin. Five groups of C57BL/6 mice (7 mice for each group): 1) Control: saline control group, 2) CP: cisplatin injury group, 3) Rg1: cisplatin + Rg1, 4) RES: cisplatin + resveratrol, 5) Rg1 + RES: cisplatin + Rg1 + resveratrol. (a) The protein levels of Beclin 1 and LC3 were determined by western blotting. GAPDH served as an internal control; (b) The mRNA levels of Beclin 1 were determined by qRT-PCR, GAPDH served as an internal control. Data are expressed as mean $\pm \mathrm{SD} .{ }^{\star} P<0.05$. 
levels of Beclin 1 in the renal tissues by qRT-PCR. The result showed that the mRNA levels of Beclin 1 in the three treatment groups were significantly downregulated than the cisplatin injury group, suggesting that Rg1 and resveratrol led to decreased autophagy which was induced by cisplatin (Figure 3(b)). It indicated that $\operatorname{Rg} 1$ and resveratrol alone or in combination reduced autophagy which was induced by cisplatin.

\section{Discussion}

Nephrotoxicity, especially AKI, as the most common and severe adverse reaction of cisplatin, is an important dose-limiting factor for cisplatin usage. Resveratrol and ginsenoside Rg1 are two important natural products. Resveratrol is a polyphenolic phytochemical that can be extracted from grapes and peanuts and other edible plants. Resveratrol has a wide range of effects, such as anti-inflammation, anti-cancer, and protection of heart, nerve and kidney. Ginsenoside Rg1 is one of the most active and abundant components in ginseng. Rg1 has good pharmacological effects on central nervous system, cardiovascular system and immune system, and has anti-inflammatory, anti-apoptotic and anti-cancer effects [13] [14]. Recently, studies have shown that both resveratrol and $\mathrm{Rg} 1$ have protective effects on the kidney, but the exact mechanism is not clear. In this study, we validated the renoprotective effects of resveratrol and Rg1. However, resveratrol showed a tendency to alleviate renal injury induced by cisplatin, but not markedly as Rg1, which may be related to the properties of resveratrol itself, such as its poor water-solubility, low bioavailability low [15] and poor stability (metabolizes rapidly and widely in rodents and humans) [16]. These may lead to the decreased efficacy of resveratrol.

The role of autophagy in renal injury is controversial. Jiang et al. showed that autophagy had a protective effect on AKI [8]. Chen et al. demonstrated the role of autophagy in $\mathrm{CaOx}$-nanocrystalline-induced cytotoxicity, using autophagy to help design promising strategies to alleviate renal injury in patients with kidney stones [17]. Huang et al. found that resveratrol protects podocyte cells from apoptosis by stimulating autophagy in a mouse model of diabetic nephropathy [18]. However, Nakagawa et al. found everolimus-induced autophagy aggravated tubular dysfunction during the recovery of renal injury [9]. Schwarze et al. demonstrated that autophagy activation of circulating proangiogenic cells (PACs) aggravated AKI in type I diabetes [19]. We found autophagy was induced by cisplatin in renal tissues of mice in a time-dependent manner (Figure 2). We considered the initial days the autophagy played a protective role against renal injury, but three days after cisplatin injection, excessively increased autophagy aggravated the renal injury.

We illustrated the resveratrol and Rg1 decreased autophagy by downregulation of the expression levels of Beclin 1 and LC3II/I which was induced by cisplatin (Figure 3). It has been found that resveratrol can inhibit cardiomyocyte death by inhibiting the ability of S6K1 to reduce DOX-induced autophagy [20]. 
Studies showed that Rg1 effectively inhibited Ang II-induced autophagy in podocyte [21], and inhibited aldosterone-induced autophagy in rat renal tubular NRK-52E cells, through AMPK/mTOR pathway [22]. Another study showed that Rg1 could improve myocardial toxicity by inhibiting autophagy and reducing endoplasmic reticulum pressure [23]. However, here were some controversial reports that resveratrol and Rg1 could induce autophagy. It was reported that resveratrol protects against spinal cord injury by activating autophagy through upregulation of SIRT1/AMPK pathway [24]. Resveratrol could induce autophagy and apoptosis in cisplatin-resistant human oral cancer CAR cells by stimulating the expression of autophagy proteins Beclin 1 and LC3-II [25]. It was found that $\operatorname{Rg} 1$ could decrease apoptosis and enhance autophagy by up-regulating the expression of Beclin 1 in H9C2 myocardial cells [26].

Besides autophagy, other mechanisms may contribute to the renoprotective effects of Rg1 and resveratrol. Rg1 could alleviate oxidative stress injury in mice with subacute renal damage induced by d-galactose [27], reduce oxidative stress by inhibiting TGF- $\beta 1 /$ Smads signaling pathway in diabetic nephropathy [6], suppress inflammation and apoptosis in LPS-induced HK-2 cells [28], and inhibit apoptotic and fibrotic process partly through inhibition of endoplasmic reticulum stress in rats with unilateral ureteral obstruction [29]. Resveratrol may alleviate renal injury induced by cisplatin via reducing apoptosis of renal tubular cells [30], reducing oxidative stress [31], inhibiting inflammation [32].

\section{Conclusion}

In conclusion, resveratrol and Rg1 alleviated the kidney damage caused by cisplatin, and reduced autophagy was involved in the renoprotective effects of resveratrol and Rg1 against cisplatin-induced AKI. This study may provide new evidence to alleviate cisplatin-induced AKI.

\section{Acknowledgements}

This work was supported by the National Natural Science Foundation of China (81602303), and the College Students' Innovative and Entrepreneurial Training Program of Yangtze University (2018190, 2018191).

\section{Conflicts of Interest}

The authors declared no competing interests.

\section{References}

[1] Miller, R.P., Tadagavadi, R.K., Ramesh, G., et al. (2010) Mechanisms of Cisplatin Nephrotoxicity. Toxins, 2, 2490-2518. https://doi.org/10.3390/toxins2112490

[2] Oh, G.S., Kim, H.J., Shen, A., et al. (2014) Cisplatin-Induced Kidney Dysfunction and Perspectives on Improving Treatment Strategies. Electrolytes \& Blood Pressure, 12, 55-65. https://doi.org/10.5049/EBP.2014.12.2.55

[3] Malhotra, A., Bath, S., Elbarbry, F.J.O.M., et al. (2015) An Organ System Approach 
to Explore the Antioxidative, Anti-Inflammatory, and Cytoprotective Actions of Resveratrol. Oxidative Medicine and Cellular Longevity, 2015, Article ID: 803971. https://doi.org/10.1155/2015/803971

[4] Kim, D.H., Jung, Y.J., Lee, J.E., et al. (2011) SIRT1 Activation by Resveratrol Ameliorates Cisplatin-Induced Renal Injury through Deacetylation of p53. American Journal of Physiology. Renal Physiology, 301, F427-F435.

https://doi.org/10.1152/ajprenal.00258.2010

[5] Wu, D., Luo, N., Wang, L., et al. (2017) Hydrogen Sulfide Ameliorates Chronic Renal Failure in Rats by Inhibiting Apoptosis and Inflammation through ROS/MAPK and NF-kappaB Signaling Pathways. Scientific Reports, 7, 455.

https://doi.org/10.1038/s41598-017-00557-2

[6] Du, N., Xu, Z., Gao, M., et al. (2018) Combination of Ginsenoside Rg1 and Astragaloside IV Reduces Oxidative Stress and Inhibits TGF-beta1/Smads Signaling Cascade on Renal Fibrosis in Rats with Diabetic Nephropathy. Drug Design, Development and Therapy, 12, 3517-3524. https://doi.org/10.2147/DDDT.S171286

[7] Periyasamy-Thandavan, S., Jiang, M., Wei, Q., et al. (2008) Autophagy Is Cytoprotective during Cisplatin Injury of Renal Proximal Tubular Cells. Kidney International, 74, 631. https://doi.org/10.1038/ki.2008.214

[8] Jiang, M., Wei, Q., Dong, G., et al. (2012) Autophagy in Proximal Tubules Protects against Acute Kidney Injury. Kidney International, 82, 1271-1283. https://doi.org/10.1038/ki.2012.261

[9] Nakagawa, S., Nishihara, K., Inui, K.I., et al. (2012) Involvement of Autophagy in the Pharmacological Effects of the mTOR Inhibitor Everolimus in Acute Kidney Injury. European Journal of Pharmacology, 696, 143-154. https://doi.org/10.1016/j.ejphar.2012.09.010

[10] Kang, R., Zeh, H.J., Lotze, M.T., et al. (2011) The Beclin 1 Network Regulates Autophagy and Apoptosis. Cell Death \& Differentiation, 18, 571-580. https://doi.org/10.1038/cdd.2010.191

[11] He, C. and Levine, B. (2010) The Beclin 1 Interactome. Current Opinion in Cell Biology, 22, 140-149. https://doi.org/10.1016/j.ceb.2010.01.001

[12] Kabeya, Y., Mizushima, N., Ueno, T., et al. (2000) LC3, a Mammalian Homologue of Yeast Apg8p, Is Localized in Autophagosome Membranes after Processing. The EMBO Journal, 19, 5720-5728. https://doi.org/10.1093/emboj/19.21.5720

[13] Zhang, Y., Zhang, Z., Wang, H., et al. (2016) Neuroprotective Effect of Ginsenoside Rg1 Prevents Cognitive Impairment Induced by Isoflurane Anesthesia in Aged Rats via Antioxidant, Anti-Inflammatory and Anti-Apoptotic Effects Mediated by the PI3K/AKT/GSK-3 $\beta$ Pathway. Molecular Medicine Reports, 14, 2778-2784. https://doi.org/10.3892/mmr.2016.5556

[14] Yu, M., Yu, X., Guo, D., et al. (2015) Ginsenoside Rg1 Attenuates Invasion and Migration by Inhibiting Transforming Growth Factor- $\beta 1$-Induced Epithelial to $\mathrm{Me}$ senchymal Transition in HepG2 Cells. Molecular Medicine Reports, 11, 3167-3173. https://doi.org/10.3892/mmr.2014.3098

[15] Catherine, A., Britton, R.G., Patel, K.R., et al. (2014) Resveratrol-Sulfates Provide an Intracellular Reservoir for Generation of Parent Resveratrol, Which Induces Autophagy in Cancer Cells. Autophagy, 10, 524-525. https://doi.org/10.4161/auto.27593

[16] Soleas, G.J., Angelini, M., Grass, L., Diamandis, E.P. and Goldberg, D.M. (2001) Absorption of Trans-Resveratrol in Rats. Methods in Enzymology, 335, 145-154. https://doi.org/10.1016/S0076-6879(01)35239-4

[17] Chen, Y., Wei, P., Meng, J., et al. (2019) Harnessing Calcium-Oxalate-(CaOx-) Na- 
nocrystal-Induced Prodeath Autophagy for Attenuating Human Renal Proximal Tubular Epithelial Cell Injury. Particle \& Particle Systems Characterization, 36, Article ID: 1900083. https://doi.org/10.1002/ppsc.201900083

[18] Huang, S.S., Ding, D.F., Chen, S., et al. (2017) Resveratrol Protects Podocytes against Apoptosis via Stimulation of Autophagy in a Mouse Model of Diabetic Nephropathy. Scientific Reports, 7, Article No. 45692.

https://doi.org/10.1038/srep45692

[19] Schwarze, K., Kribben, A., Ritter, O., et al. (2018) Autophagy Activation in Circulating Proangiogenic Cells (PACs) Aggravates AKI in Type I Diabetes Mellitus. The American Journal of Physiology: Renal Physiology, 315, F1139-F1148. https://doi.org/10.1152/ajprenal.00502.2017

[20] Xu, X.M., Chen, K., et al. (2012) Resveratrol Attenuates Doxorubicin-Induced Cardiomyocyte Death via Inhibition of p70 S6 Kinase 1-Mediated Autophagy. The Journal of Pharmacology and Experimental Therapeutics, 341, 183-195.

https://doi.org/10.1124/jpet.111.189589

[21] Mao, N., Tan, R.Z., Wang, S.Q., et al. (2016) Ginsenoside Rg1 Inhibits Angiotensin II-Induced Podocyte Autophagy via AMPK/mTOR/PI3K Pathway. Cell Biology International, 40, 917-925. https://doi.org/10.1002/cbin.10634

[22] Wang, L., Mao, N., Tan, R.Z., et al. (2015) Ginsenoside Rg1 Reduces Aldosterone-Induced Autophagy via the AMPK/mTOR Pathway in NRK-52E Cells. International Journal of Molecular Medicine, 36, 518-526. https://doi.org/10.3892/ijmm.2015.2242

[23] Xu, Z.M., Li, C.B., Liu, Q.L., et al. (2018) Ginsenoside Rg1 Prevents Doxorubicin-Induced Cardiotoxicity through the Inhibition of Autophagy and Endoplasmic Reticulum Stress in Mice. International Journal of Molecular Sciences, 19, pii: E3658. https://doi.org/10.3390/ijms19113658

[24] Zhao, H., Chen, S., Gao, K., et al. (2017) Resveratrol Protects against Spinal Cord Injury by Activating Autophagy and Inhibiting Apoptosis Mediated by the SIRT1/AMPK Signaling Pathway. Neuroscience, 348, 241-251. https://doi.org/10.1016/j.neuroscience.2017.02.027

[25] Chang, C.H., Lee, C.Y., Lu, C.C., et al. (2017) Resveratrol-Induced Autophagy and Apoptosis in Cisplatin-Resistant Human Oral Cancer CAR Cells: A Key Role of AMPK and Akt/mTOR Signaling. International Journal of Oncology, 50, 873-882. https://doi.org/10.3892/ijo.2017.3866

[26] Li, D., Wang, J., Hou, J., et al. (2016) Ginsenoside Rg1 Protects Starving H9c2 Cells by Dissociation of Bcl-2-Beclin1 Complex. BMC Complementary Medicine and Therapies, 16, 146-158. https://doi.org/10.1186/s12906-016-1112-2

[27] Fan, Y., Xia, J., Jia, D., et al. (2016) Mechanism of Ginsenoside Rg1 Renal Protection in a Mouse Model of d-Galactose-Induced Subacute Damage. Pharmaceutical Biology, 54, 1815-1821. https://doi.org/10.3109/13880209.2015.1129543

[28] Ni, X.J., Xu, Z.Q., Jin, H., et al. (2017) Ginsenoside Rg1 Protects Human Renal Tubular Epithelial Cells from Lipopolysaccharide-Induced Apoptosis and Inflammation Damage. Brazilian Journal of Medical and Biological Research, 51, e6611. https://doi.org/10.1590/1414-431x20176611

[29] Li, S.S., Ye, J.M., Deng, Z.Y., et al. (2015) Ginsenoside-Rg1 Inhibits Endoplasmic Reticulum Stress-Induced Apoptosis after Unilateral Ureteral Obstruction in Rats. Renal Failure, 37, 890-895. https://doi.org/10.3109/0886022X.2015.1015427

[30] Zhang, R., Yin, L., Zhang, B., et al. (2018) Resveratrol Improves Human Umbilical Cord-Derived Mesenchymal Stem Cells Repair for Cisplatin-Induced Acute Kidney 
Injury. Cell Death \& Disease, 9, 965.

[31] Valentovic, M.A., Ball, J.G., Brown, J.M., et al. (2014) Resveratrol Attenuates Cisplatin Renal Cortical Cytotoxicity by Modifying Oxidative Stress. Toxicology in $\mathrm{Vi}$ tro, 28, 248-257. https://doi.org/10.1016/j.tiv.2013.11.001

[32] Amaral, C.L.D., Francescato, H.D.C., Coimbra, T.M., et al. (2008) Resveratrol Attenuates Cisplatin-Induced Nephrotoxicity in Rats. Archives of Toxicology, 82, 363-370. https://doi.org/10.1007/s00204-007-0262-x 